\title{
磁性多壁碳纳米管对水中三种硝基咪唑类药物的吸附行为
}

\author{
任晓东 $a, b$ 熊振湖*,a,b \\ ( ${ }^{a}$ 天津城市建设学院 环境与市政工程学院 天津 300384) \\ $\left({ }^{b}\right.$ 天津城市建设学院 天津市水质科学与技术重点实验室 天津 300384)
}

\begin{abstract}
摘要 在制备磁性多壁碳纳米管(MWCNTs)并且对其表征的基础上，将磁性 MWCNTs 用于吸附水溶液中的三种硝基 咪唑类药物(甲硝唑、奥硝唑、替硝唑). 研究了溶液 $\mathrm{pH}$ 值、吸附剂量、吸附时间和温度对吸附过程的影响. 结果表明, 当磁性 MWCNTs 投加量为 $5 \mathrm{~g} \cdot \mathrm{L}^{-1}$, 溶液 $\mathrm{pH}$ 值为 7 , 吸附时间为 $300 \mathrm{~min}$ 时, 甲硝唑、奥硝唑、替硝唑的去除率分别 达到 92.86\%, 94.44\%, 94.91\%. 吸附动力学分析证实准二级模型准确地反映了吸附过程的动力学, 而 Freundlich 模型很 好地描述了不同温度下的吸附等温线. 吸附过程的总速度受到外部质量传递和粒子内扩散的影响, 但主导因素是粒子 内扩散. 吸附机理可能是由 MWCNTs 和硝基咪唑类化合物之间的静电引力及 $\pi-\pi$ 堆积作用产生的. 对热力学参数 $\left(\Delta H^{0}, \Delta S^{0}, \Delta G^{0}\right)$ 的计算揭示, 在实验的温度范围, 硝基咪唑类药物的吸附是自发、放热的物理过程. 最后考察了吸附材 料中 $\mathrm{Fe}$ 的溢出情况, 发现在中性 $\mathrm{pH}$ 下 $\mathrm{Fe}$ 的溢出仅是 $0.96 \mathrm{mg} \cdot \mathrm{L}^{-1}$, 表明磁性 MWCNTs 在中性水体中是相当稳定的. 关键词 磁性多壁碳纳米管; 硝基咪唑类药物; 吸附; 动力学; 热力学
\end{abstract}

\section{Adsorption Behavior of Three Nitroimidazoles in Aqueous Solutions to Magnetic-modified Multi-walled Carbon Nanotubes}

\author{
Ren, Xiaodong ${ }^{a, b} \quad$ Xiong, Zhenhu ${ }^{*, a, b}$
}

( ${ }^{a}$ Collage of Environmental and Municipal Engineering, Tianjin Institute of Urban Construction, Tianjin 300384, China)

$\left({ }^{b}\right.$ Collage of Environmental and Municipal Engineering, Tianjin Key Laboratory of Water Quality Science and Technology, Tianjin 300384, China)

\begin{abstract}
The magnetic multi-walled carbon nanotubes (MWCNTs) were synthesized by wet chemical treatments. Firstly, an amount of $1.0 \mathrm{~g}$ of purified MWCNTs was suspended in $200 \mathrm{~mL}$ of mixed solution containing $1.7 \mathrm{~g}$ of ammonium ferrous sulfate and $2.5 \mathrm{~g}$ of ammonium ferric sulfate, which was followed by a slow addition of $10.0 \mathrm{~mL}$ of $8 \mathrm{~mol} \cdot \mathrm{L}^{-1} \mathrm{NH}_{4} \mathrm{OH}$ solution at constant temperature of $50{ }^{\circ} \mathrm{C}$, under nitrogen atmosphere, with the aid of ultrasonic stirring for $10 \mathrm{~min}$. The $\mathrm{pH}$ of the final mixture was controlled in the range of $11 \sim 12$. The reaction was allowed to continue for $30 \mathrm{~min}$, which results in the color of the suspension changing from black to brown. After the completion of the reaction, the suspension was allowed to cool at room temperature. The magnetic MWCNTs was isolated from the mixture by a permanent magnet, afterwards dried under vacuum. The size and morphology of the synthesized magnetic MWCNTs were characterized by transmission electron microscopy, X-ray diffraction, Fourier transform infrared spectroscopy and vibrating sample magnetometer. The magnetic MWCNTs was used to adsorbing the three nitroimidazoles, - metronidazole, ornidazole and tinidazole - in aqueous solutions. The adsorption kinetic (or isotherms) experiments were carried out by adding $250 \mathrm{mg}$ of magnetic MWCNTs to a 50 $\mathrm{mL}$ flask which containing a solution made up of one or three nitroimidazoles with known initial concentration $\left(10 \mathrm{mg} \cdot \mathrm{L}^{-1}\right)$ at natural $\mathrm{pH}$, then agitating in a temperature-controlled shaker at $(25 \pm 1){ }^{\circ} \mathrm{C}$ for different time intervals $(1 \sim 480 \mathrm{~min})$. The effect of $\mathrm{pH}$ was performed by dispersion of $250 \mathrm{mg}$ of magnetic MWCNTs in $50 \mathrm{~mL}$ aqueous solution which containing 10 $\mathrm{mg} \cdot \mathrm{L}^{-1}$ of three nitroimidazoles of each. The results showed that the removal of metronidazole, ornidazole and tinidazole reached $92.86 \%, 94.44 \%, 94.91 \%$ respectively when the dosage of magnetic MWCNTs was $5 \mathrm{~g} \cdot \mathrm{L}^{-1}$, the $\mathrm{pH}$ values was 7 and the time of adsorption was $300 \mathrm{~min}$. Adsorption kinetic analysis demonstrated that pseudo-second order model could interpret the kinetics of the adsorption process, and Freundlich model could better explains the isotherm for adsorption process under different temperatures. The overall adsorption rate appeared to be influenced by both the external mass transfer and the intraparticle diffusion, and mainly governed by the intraparticle diffusion. The adsorption mechanism could be explained by electrostatic attraction between MWCNTs and nitroimidazoles, and by $\pi-\pi$ stacking interaction. The obtained thermodynamic parameters $\left(\Delta H^{0}, \Delta S^{0}, \Delta G^{0}\right)$, calculated from adsorption process revealed that 1) $\Delta G^{0}<0$ indicated that the adsorption of nitroimidazoles on magnetic MWCNTs was spontaneous; 2) $\Delta G^{0}$ indicated that the adsorption is physical process; 3) $\Delta H^{0}<0$ indicated that the absorption reaction was exothermic and that low temperatures were favorable for adsorption, and 4$) \Delta S^{0}>0$ indicated that the adsorption process was an entropy enhancing process in the experimental temperature range. Finally, the leaching of $\mathrm{Fe}$ from adsorbent into the treated water was tested, which found that the leaching of Fe was as low as
\end{abstract}

* E-mail: zhenhu.xiong@126.com

Received November 13, 2012; published February 6, 2013.

Project supported by the National Natural Science Foundation of China (No. 50878138).

项目受国家自然科学基金(No. 50878138)资助. 
$0.96 \mathrm{mg} \cdot \mathrm{L}^{-1}$. It indicated that the magnetic MWCNT was very stable in neutral aqueous solution.

Keywords magnetic multi-walled carbon nanotubes; nitroimidazoles; adsorption; kinetics; thermodynamics

\section{1 引言}

甲硝唑(Metronidazole)、替硝唑(Tinidazole)和奥硝 唑(Ornidazole)是第一、二和三代硝基咪唑类药物的典型 代表物 ${ }^{[1 ~ 3]}$, 由于它们强力的抗厌氧菌和抗原虫活性并 且价格低廉, 目前仍被广泛用于治疗人与动物的厌氧菌 感染和防治各种原虫病. 三种药物的抗厌氧菌机制均是 通过其分子中的硝基在无氧环境中还原成氨基, 或者通 过形成自由基与细胞成分相互作用, 从而导致微生物死 亡. 然而, 大量研究证实硝基咪唑类药物具有细胞诱 变、动物致癌等潜在的严重危害性 ${ }^{[4,5]}$. 由于硝基咪唑类 药物难以被生物降解 ${ }^{[6,7]}$, 在天然水体和污水处理厂出 水中已检测到它们的存在 ${ }^{[8,9]}$, 而长期的积累就会对人 类健康和生态系统的安全构成威胁.

多壁碳纳米管(Multi Carbon Nanotubes, MWCNTs) 是一种具有高比表面积和大微孔体积的新型碳材料, 表 面的 $\pi$ 电子结构使其对于芳香化合物表现出很强的吸附 能力, 被广泛用于吸附水中的有机污染物和重金属离 子 ${ }^{[10,11]}$. 目前尚没有采用 MWCNTs 同时清除水中硝基 咪唑类药物的报道, 也不知晓这类化合物在 MWCNTs 上的吸附动力学和热力学特性.

本研究在制备 $\mathrm{Fe}_{3} \mathrm{O}_{4}$ 纳米粒子基础上, 制备了磁性 MWCNTs, 系统地研究了硝基咪唑类药物在 MWCNTs 上的吸附动力学、等温线和热力学, 而且通过外磁场很 容易地将吸附剂从水介质中分离出来. 本研究目的是了 解硝基咪唑类药物与 MWCNTs 之间的相互作用, 优化 MWCNTs 去除水中硝基咪唑类药物的条件. 硝基咪唑 类药物在 MWCNTs 上吸附行为的研究为制备有效去除 水中硝基咪唑类药物的吸附材料提供了实验依据, 而且 为硝基咪唑类药物的在线监测以及相关电化学传感器 的制备提供了理论基础.

\section{2 结果与讨论}

\section{1 试剂与材料}

MWCNTs 购自中国科学院成都有机化学有限公 司, 其内径为 $5 \sim 10 \mathrm{~nm}$, 外径为 $10 \sim 20 \mathrm{~nm}$, 长度约为 $50 \mu \mathrm{m}$, 比表面积约为 $200 \mathrm{~m}^{2} \cdot \mathrm{g}^{-1}$. 所用的其它试剂为分 析级, 来自天津江天化学品有限公司 (天津, 中国), 全 部实验都使用娃哈哈公司提供的纯净水. 所有的玻璃器 皿用硝酸浸泡 $12 \mathrm{~h}$, 最后在使用前用纯净水淋洗三次.

图 1 给出了本研究涉及的三种硝基咪唑类化合物的 结构式. 药物的本体溶液是其粉末溶解在纯净水中制备 而成, 并且用纯净水将本体溶液稀释到所需浓度, 以此 方式制备不同初始浓度的药物溶液.

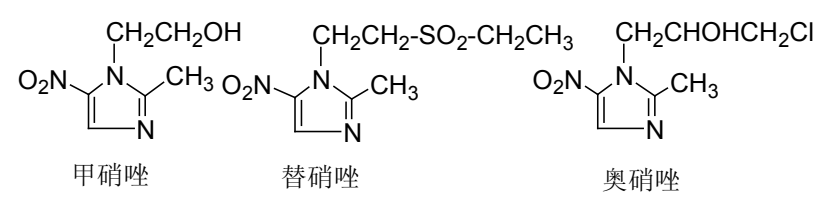

图 1 三种硝基咪唑类药物的结构

Figure 1 The structures of the three nitroimidazole pharmaceuticals

\section{2 磁性多壁碳纳米管的制备}

综合文献[12 14]的方法制备磁性 MWCNTs. 称取 $1.5 \mathrm{~g}$ MWCNTs 加入到 $250 \mathrm{~mL}$ 四口瓶中, 再加入 150 $\mathrm{mL}$ 浓硝酸, 机械搅拌下油浴升温 $60{ }^{\circ} \mathrm{C}$, 在此温度下恒 速摚拌 $12 \mathrm{~h}$, 反应结束后冷却至室温, 用大量去离子水 洗涤至中性. 在 $100{ }^{\circ} \mathrm{C}$ 干燥箱中干燥 $8 \mathrm{~h}$, 冷却后放置在 干燥器中备用.

称取 $1.0 \mathrm{~g}$ 纯化后的 MWCNTs, 悬浮在由 $1.7 \mathrm{~g}(4.33$ $\mathrm{mmo1})\left(\mathrm{NH}_{4}\right)_{2} \mathrm{Fe}\left(\mathrm{SO}_{4}\right)_{2} \cdot 6 \mathrm{H}_{2} \mathrm{O}$ 与 $2.51 \mathrm{~g}(8.66 \mathrm{mmol})$ $\mathrm{NH}_{4} \mathrm{Fe}\left(\mathrm{SO}_{4}\right)_{2} \cdot 12 \mathrm{H}_{2} \mathrm{O}$ 溶于 $200 \mathrm{~mL}$ 纯净水构成的混合溶 液中, 超声 $10 \mathrm{~min}$, 氮气保护下逐滴加入 $10 \mathrm{~mL} 8 \mathrm{~mol} \cdot$ $\mathrm{L}^{-1}$ 的氨水溶液使体系 $\mathrm{pH}$ 值保持在 $11 \sim 12$, 于 $50{ }^{\circ} \mathrm{C}$ 恒 温和 $300 \mathrm{r} \cdot \mathrm{min}^{-1}$ 恒速摚拌下反应 $30 \mathrm{~min}$. 反应完成后悬 浮液由黑色变为褐色. 反应完成后用磁铁将磁性 MWCNTs 从悬浮液中分离出来, 分别用去离子水和无 水乙醇洗涤 3 次, $40{ }^{\circ} \mathrm{C}$ 真空干燥后放入干燥器中备用.

\section{3 表征}

水溶液中甲硝唑、奥硝唑、替硝唑的浓度由一台 T6 紫外可见分光光度计(北京普析通用仪器有限责任公 司) 测定. 使用一台傅里叶变换红外光谱仪 (FTIR, NEXUS-5670, Nicolet, America)检测红外光谱数据. 磁 性 MWCNTs 纳米微粒的大小、形态和结构由一台透射 电子显微镜(TEM, Philips, CM10, 100 KV)测定, 而它的 $\mathrm{X}$-射线衍射图谱由一台 $\mathrm{D} / \mathrm{max} 2550 \mathrm{X}$ 型 $\mathrm{X}$ 射线衍射仪 (RigaKu, Japan)进行分析.

固体的零电荷点 $\left(\mathrm{pH}_{\mathrm{PZC}}\right)$ 可给出吸附剂与 $\mathrm{pH}$ 相关的 特性 ${ }^{[15]}$. 在一系列 $100 \mathrm{~mL}$ 锥形瓶中, 置入 $45 \mathrm{~mL}$ 浓度 为 $0.1 \mathrm{~mol} \cdot \mathrm{L}^{-1}$ 的 $\mathrm{NaCl}$ 溶液. 通过加入 $0.1 \mathrm{~mol} \cdot \mathrm{L}^{-1} \mathrm{HCl}$ 或 $0.1 \mathrm{~mol} \cdot \mathrm{L}^{-1} \mathrm{NaOH}$ 将溶液的初始 $\mathrm{pH}$ 值 $\left(\mathrm{pH}_{\mathrm{i}}\right)$ 从 2.0 调 节到 10.0. 加入 $\mathrm{NaCl}$ 溶液使每个雉形瓶中溶液总体积 精确达到 $50 \mathrm{~mL}$. 然后, 在每个瓶子中加入 $0.25 \mathrm{~g}$ 磁性 MWCNTs, 并且将混合物在 $150 \mathrm{r} \bullet \mathrm{min}^{-1}$ 下振摇. 在 $48 \mathrm{~h}$ 之后, 检测溶液的最终 $\mathrm{pH}$ 值 $\left(\mathrm{pH}_{\mathrm{f}}\right)$. 将初始和最终 $\mathrm{pH}$ 值 之间的差值对 $\mathrm{pH}_{\mathrm{i}}$ 绘图, 所得曲线与横坐标的交点为 $\Delta \mathrm{pH}=0$ 点, 此点即为 $\mathrm{pH}_{\mathrm{PZC}}$.

\section{4 间歇吸附实验}

在 $50 \mathrm{~mL}$ 烧瓶中依次加入 $250 \mathrm{mg}$ 磁性 MWCNTs 
和一定初始浓度的甲硝唑、奥硝唑、替硝唑混合液溶, 必 要时用 $0.1 \mathrm{~mol} \cdot \mathrm{L}^{-1} \mathrm{HCl}$ 或 $0.1 \mathrm{~mol} \cdot \mathrm{L}^{-1} \mathrm{NaOH}$ 调节悬浮 液 $\mathrm{pH}$. 将盛有混合溶液的烧瓶置于一台 HZQ-QS 振荡 器中, 以 $150 \mathrm{r} \cdot \mathrm{min}^{-1}$ 的速率振摇, 在规定时间从溶液中 取样, 外部磁铁分离出磁性 MWCNTs, 分别在 277、285 和 $317 \mathrm{~nm}$ 的甲硝唑、奥硝唑和替硝唑最大吸收波长下, 用 UV-Vis 分光光度计测定样品溶液中三种硝基咪唑类 药物的残留浓度, 用下列方程式计算每个药物的吸附百 分数(Ads. $\%)$.

$$
\text { Ads. } \%=\left[\frac{\left(C_{0}-C_{t}\right)}{C_{0}}\right] \times 100
$$

式中, $C_{0}$ 和 $C_{t}$ 分别代表药物的初始浓度(吸附前)与最终 浓度(吸附后), 单位为 $\mathrm{mg} \cdot \mathrm{L}^{-1}$. 所有的测试都进行三次, 以确保结果的可重复性; 以三次测量的平均值为准. 所 有的实验均在室温下进行.

在 288 308 K 温度范围, 在 $50 \mathrm{~mL}$ 烧瓶中加入一 系列 $250 \mathrm{mg}$ 磁性 MWCNT 和 $50 \mathrm{~mL} 10 \mathrm{mg} \cdot \mathrm{L}^{-1}$ 的药物 溶液, 进行动力学吸附实验. 在规定的时间范围内取出 适量分数样品, 测定各种硝基咪唑类药物的浓度. 使用 常规速率方程计算速度常数.

在 5 到 $15 \mathrm{mg} \cdot \mathrm{L}^{-1}$ 范围的硝基咪唑类药物初始浓度 进行吸附等温线研究, 吸附剂量保持为常数 $(250 \mathrm{mg})$, 实验温度分别控制在 288,298 和 $308 \mathrm{~K}$. 记录每个温度 下吸附过程的热力学参数.

\section{5 泄露试验}

为了评价磁性 MWCNTs 的稳定性, 在不同 $\mathrm{pH}$ 下研 究了 M-MWCNTs 上铁离子的溢出问题. 将 $250 \mathrm{mg}$ 磁性 MWCNTs 分散在 $\mathrm{pH}$ 范围在 2.0 到 10.0 的 $50 \mathrm{~mL}$ 水溶 液中, 于 $(25 \pm 1){ }^{\circ} \mathrm{C}$ 的控温摇床上振摇 $300 \mathrm{~min}$. 由一台 AAnalyst 800 原子吸收光谱仪(Perkin-Elmer)测定上清液 中溢出的 $\mathrm{Fe}$ 浓度.

\section{3 讨论}

\section{1 磁性多壁碳纳米管的表征}

图 2(a)是 MWCNTs 的 TEM 图像, 可以发现相对较 长的 MWCNTs 相互纠缠在一起. 在图 2(b) 中可见 $\mathrm{Fe}_{3} \mathrm{O}_{4}$ 纳米颗粒包覆在 MWCNTs 表面, 而且 MWCNTs 内部充 斥着 $\mathrm{Fe}_{3} \mathrm{O}_{4}$ 纳米颗粒, 形成 $\mathrm{MWCNTs} / \mathrm{Fe}_{3} \mathrm{O}_{4}$ 纳米复合物. $\mathrm{Fe}^{2+}$ 与 $\mathrm{Fe}^{3+}$ 进入到 MWCNTs 的内部而原位生成 $\mathrm{Fe}_{3} \mathrm{O}_{4}$ 的原因是 MWCNTs 内孔中的毛细管力足以克服水的表 面张力 $\left(72 \mathrm{mN} \cdot \mathrm{m}^{-1}\right)^{[16]}$. 另外, 硝酸的氧化处理使 MWCNTs 被切断, 形成开口端和表面功能基团, 这也 有助于铁离子进入 MWCNTs 的内部及其在表面的吸附.

图 3 给出了 MWCNTs、 $\mathrm{Fe}_{3} \mathrm{O}_{4}$ 和磁性 MWCNTs 的 $\mathrm{X}$-射线衍射(XRD)图谱. 图 $3 \mathrm{a}$ 中, 在 $2 \theta=25.91^{\circ}$ 处, 是 MWCNTs 的六方石墨结构 ${ }^{[17]}$. 如图 3(c)所示, 在负载

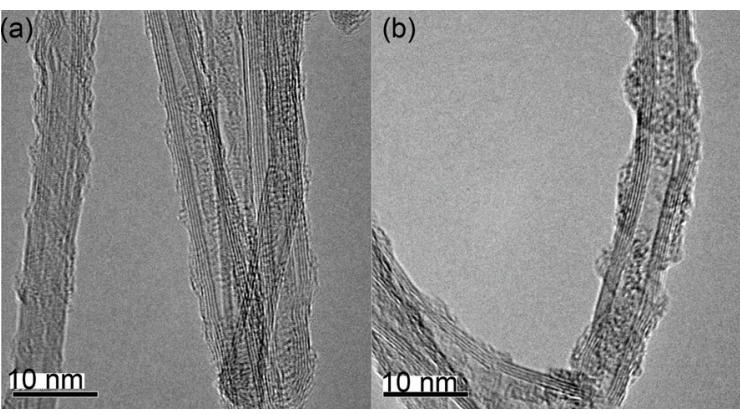

图 2 (a) MWCNTs 和(b)磁性 MWCNTs 的 TEM 图

Figure 2 TEM images of (a) MWCNTs and (b) magnetic MWCNTs

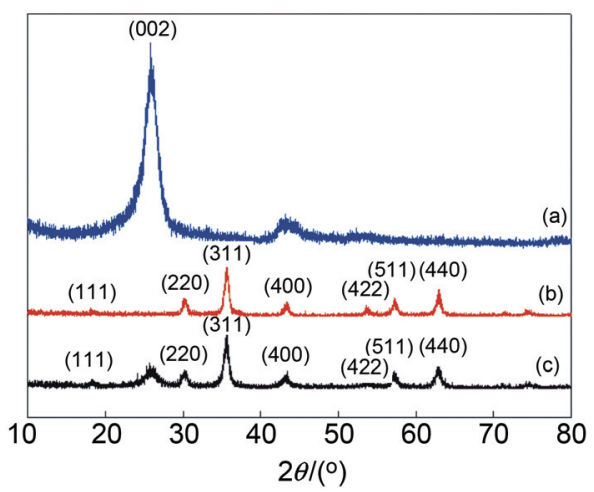

图 3 (a) MWCNTs, (b) $\mathrm{Fe}_{3} \mathrm{O}_{4}$ 和(c)磁性 MWCNTs 的 XRD 模式

Figure 3 X-ray diffraction patterns of (a) MWCNTs, (b) $\mathrm{Fe}_{3} \mathrm{O}_{4}$ and (c) magnetic MWCNTs

$\mathrm{Fe}_{3} \mathrm{O}_{4}$ 之后, 除了 MWCNTs 的衍射峰之外, 在 $2 \theta=$ $18.2^{\circ}, 30.2^{\circ}, 35.6^{\circ}, 43.3^{\circ}, 53.7^{\circ}, 57.2^{\circ}, 62.8^{\circ}$ 处出现了新 峰, 为磁铁矿 $\left(\mathrm{Fe}_{3} \mathrm{O}_{4}\right)$ 或赤铁矿 $\left(\mathrm{Fe}_{2} \mathrm{O}_{3}\right)$ 的标准峰 ${ }^{[18]}$, 表明 在磁性 MWCNTs 中 $\mathrm{Fe}_{3} \mathrm{O}_{4}$ 和 MWCNTs 的共同存在.

图 4 给出了 $\mathrm{Fe}_{3} \mathrm{O}_{4} 、 \mathrm{MWCNTs}$ 、磁性 MWCNTs 的 FTIR 谱. 在图 4(a) $\mathrm{Fe}_{3} \mathrm{O}_{4}$ 的 FTIR 中, 在 $583 \mathrm{~cm}^{-1}$ 和 403 $\mathrm{cm}^{-1}$ 处出现的峰分别归属于 $\mathrm{Fe}_{3} \mathrm{O}_{4}$ 四面体和八面体点位 的 $v_{(\mathrm{Fe}-\mathrm{O})}$ 特有吸收 ${ }^{[19]}$. 比较图 4(b) 与图 4(c), 虽然 MWCNTs 和磁性 MWCNTs 的吸收峰几乎等同, 但磁性 MWCNTs 中出现了 $\mathrm{Fe}_{3} \mathrm{O}_{4}$ 的特征峰, 表明在 $\mathrm{Fe}_{3} \mathrm{O}_{4}$ 与 MWCNTs 之间存在相互作用. 在图 4(c)中, 磁性 MWCNTs 的特征吸附峰分别出现在 $3454 \mathrm{~cm}^{-1}(\mathrm{O}-\mathrm{H}$ 伸缩)和 $1647 \mathrm{~cm}^{-1}$ ( $\mathrm{C}=\mathrm{O}$ 伸缩), 意味着 MWCNTs 表面 具备了一定量的含氧官能团, 为磁性颗粒的成核与生长 提供了一些活性的针定位点. 图(a) (c)中在 $2360 \mathrm{~cm}^{-1}$ 附近都出现了吸收峰, 这可能来自空气中二氧化碳 $\mathrm{C}=$ $\mathrm{O}$ 键的特征吸收 ${ }^{[20]}$.

采用振动样品磁强计(VSM)研究了磁性 MWCNTs 的磁性质. 图 5 显示样品的磁化强度曲线无磁滞现象并 且原点对称, 表明样品是超顺磁性的 ${ }^{[21]}$, 这有利于磁性 分离和重复使用. 磁性 MWCNTs 在室温下的饱和磁性 $\left(M_{\mathrm{s}}\right)$ 值是 $39.15 \mathrm{emu}^{-1} \mathrm{~g}^{-1}$. 与 $\mathrm{Fe}_{3} \mathrm{O}_{4}$ 饱和磁性比较, 磁性 MWCNTs 的饱和磁响应值有所下降 ${ }^{[22]}$, 但比表面积从 


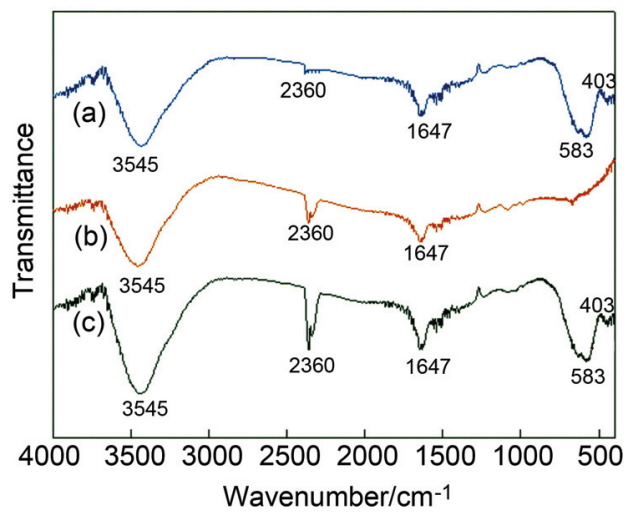

图 $4 \mathrm{Fe}_{3} \mathrm{O}_{4}(\mathrm{a})$, MWCNTs (b)和磁性 MWCNTs (c)的傅里叶变换红外 光谱

Figure 4 FTIR spectra of $\mathrm{Fe}_{3} \mathrm{O}_{4}$ (a), MWCNTs (b) and magnetic MWCNTs (c)

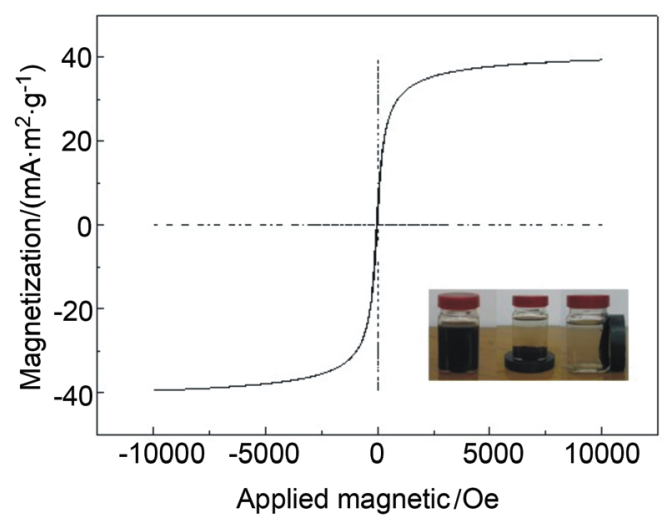

图 5 室温下磁性 MWCNTs 的 VSM 磁化强度曲线与外磁场存在下水 中磁性 MWCNTs 的图像

Figure 5 Magnetization curves of magnetic MWCNTs obtained by VSM at room temperature and a photograph of magnetic MWCNTs in the water in the presence of an external magnetic field

$307.8 \mathrm{~m}^{2} / \mathrm{g}$ 增加到 $402.8 \mathrm{~m}^{2} / \mathrm{g}^{[16]}$. 这是可预料的, 原因是 $\mathrm{Fe}_{3} \mathrm{O}_{4}$ 纳米粒子分布在 MWCNTs 的孔内与表面, MWCNTs 可在一定程度屏蔽磁性, 但磁化过程中的强 氧化使得 MWCNTs 比表面积增加. 实际上, 这种磁化 强度的纳米颗粒具有足够的磁响应性来满足迅速磁性 分离的需要, 如图 5 插图所示当外磁场存在时黑色的磁 性颗粒被吸引到小瓶的侧壁与瓶底, 伴随着溶液变得透 明与清澈.

\section{2 溶液 $\mathrm{pH}$ 的影响}

三种硝基咪唑化合物混合溶液中的每种物质的初 始浓度固定在 $10 \mathrm{mg} \cdot \mathrm{L}^{-1}$, 将磁性 MWCNTs 投加量定为 $5 \mathrm{~g} \cdot \mathrm{L}^{-1}$, 反应时间为 $6 \mathrm{~h}$, 反应温度为 $298 \mathrm{~K}$, 测试在不 同 $\mathrm{pH}$ 值下的吸附行为.

吸附结果表明(图 6), 随溶液 $\mathrm{pH}$ 增大, 甲硝唑、奥 硝唑、替硝唑的去除率都大幅度增加, 当 $\mathrm{pH}$ 为中性时 达到最大值 $(>90 \%)$. 若 $\mathrm{pH}$ 继续增大至碱性, 则三种药 物的去除率急剧下降. 低 $\mathrm{pH}$ 下三种药物去除率低是有

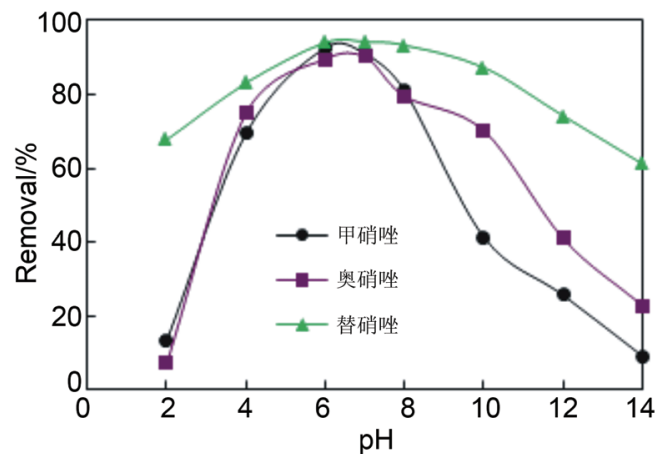

图 6 不同 $\mathrm{pH}$ 值对甲硝唑、奥硝唑、替硝唑去除率的影响

Figure 6 The effect of solution $\mathrm{pH}$ on the removal of three nitroimidazole pharmaceuticals

规律的. 咪唑环中有两个氮原子, 虽然二者都呈现 $\mathrm{sp}^{2}$ 杂化态，但分属不同的类型，一种是吡咯类型的氮，另 一种是吡啶类型的氮 ${ }^{[23]}$. 吡啶氮上的未共用电子对可 接受质子(具碱性), 而且低 $\mathrm{pH}$ 下水溶液中大量的水合 质子 $\left(\mathrm{H}_{3} \mathrm{O}^{+}\right)$可中和吡啶氮的碱性, 使得吸附质与 MWCNTs 表面羧基( $-\mathrm{COOH}$ )的作用变得困难. 另一方 面, 与一 $\mathrm{COOH}$ 比较, $\mathrm{H}_{3} \mathrm{O}^{+}$更容易与吡啶氮发生酸碱作 用, 进而屏蔽了吸附剂与吸附质之间的静电引力. 随 $\mathrm{pH}$ 值增加至中性, 溶液中 $\mathrm{H}_{3} \mathrm{O}^{+}$浓度不断下降, 吡啶氮 上的未共用电子对逐渐摆脱 $\mathrm{H}_{3} \mathrm{O}^{+}$的束缚, 磁性 MWCNTs 表面正电荷密度也在下降，导致吸附剂与芳 香性咪唑环之间的静电引力变强, 使三种药物的吸附量 迅速增加. 在中性 $\mathrm{pH}$ 下, MWCNTs 与芳香性咪唑环之 间的 $\pi-\pi$ 堆积作用也促进了二者的结合. 在 $\mathrm{pH}>7$, 溶 液中不断增加的羟基 $\left(\mathrm{OH}^{-}\right)$负离子使磁性 MWCNTs 上 的- $\mathrm{COOH}$ 基团离子化, 增加了表面负电荷密度, 过量 的 $\mathrm{OH}^{-}$还促使硝基咪唑类药物上吡啶氮呈现碱性, 负电 荷的磁性 MWCNTs 与同为负离子形态的甲硝唑、奥硝 唑、替硝唑发生排斥，从而抵消了 $\pi-\pi$ 堆积作用的引力, 使三种药物的去除率迅速下降.

上述现象还可以通过零电荷点 $\left(\mathrm{pH}_{\mathrm{PZC}}\right)$ 进行解释. 零电荷点 $\left(\mathrm{pH}_{\mathrm{PZC}}\right)$ 反映的表面电荷被定义为 zeta 电位为 零的点 ${ }^{[15]}$. 当 $\mathrm{pH}<\mathrm{pH}_{\mathrm{PZC}}$, 表面电荷为正, 而当 $\mathrm{pH}>$ $\mathrm{pH}_{\mathrm{PZC}}$ 时, 表面电荷为负. 在本实验中测得磁性 MWCNTs 的 $\mathrm{pH}_{\mathrm{PZC}}$ 约是 6.8(图 7). 因此, 当溶液 $\mathrm{pH}$ 低 于 $\mathrm{pH}_{\mathrm{PZC}}$ 时, 磁性 MWCNTs 表面电荷为正. 在这个情况 下, $\mathrm{H}_{3} \mathrm{O}^{+}$的竞争效应及其三种药物(吡啶氮与 $\mathrm{H}_{3} \mathrm{O}^{+}$的作 用使其带有部分的正电荷)和磁性 MWCNTs 表面正电荷 活性吸附位点之间的静电排斥将导致药物吸附容量的 降低，而溶液 $\mathrm{pH}$ 高于 $\mathrm{pH}_{\mathrm{PZC}}$ 时磁性 MWCNTs 的表面呈 现部分的负电荷. 由此, 负性活性吸附位点与咪唑环吡 啶氮上未共用电子对之间的静电排斥也不利于药物的 吸附。

\section{3 吸附剂用量的影响}

$50 \mathrm{~mL}$ 溶液中混合药物的浓度为 $10 \mathrm{mg} \cdot \mathrm{L}^{-1}$, 在 $\mathrm{pH}$ 


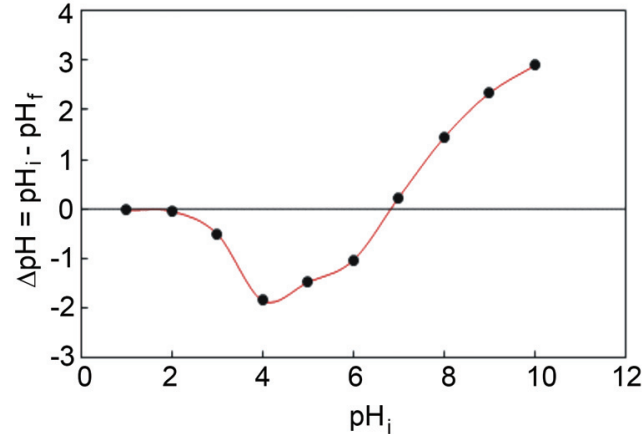

图 7 固体加入法测定的磁性 MWCNTs 的零电荷点

Figure 7 The point of zero charge of the magnetic MWCNTs determined by the solid addition method

7.0 与室温下、将磁性 MWCNTs 的量从 $150 \mathrm{mg}$ 变化至 $270 \mathrm{mg}$, 研究了药物的去除率与磁性 MWCNTs 量的关 系. 结果如图 8 所示. 显然, 三种药物的去除率随磁性 MWCNTs 量的增加而增大, 原因是这种情况下吸附剂 表面可利用的吸附位点较多. 当磁性 MWCNTs 的量超 过 $5 \mathrm{~g} \cdot \mathrm{L}^{-1}$ 时, 甲硝唑、奥硝唑、替硝唑的去除率达到平 衡，依次为 $92.37 \%, 90.70 \%, 90.28 \%$. 因此, 将磁性 MWCNTs 投加量定为 $5 \mathrm{~g} \cdot \mathrm{L}^{-1}$.

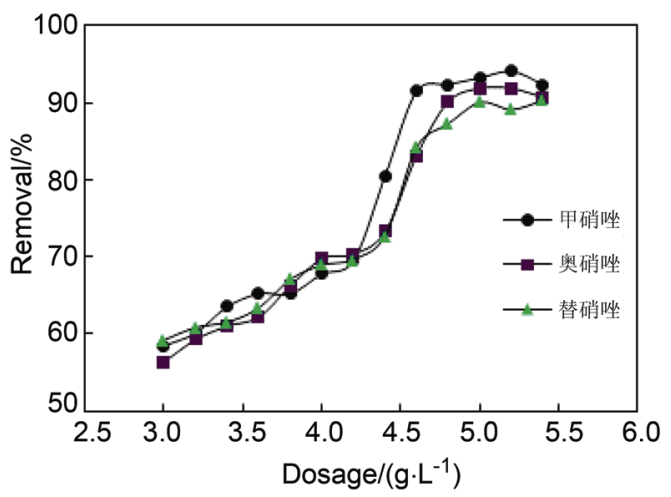

图 8 不同磁性 MWCNTs 的量对三种药物的去除率. 条件: pH 7.0, 药物溶液为 $50 \mathrm{~mL}$, 浓度为 $10 \mathrm{mg} \cdot \mathrm{L}^{-1}$ 、振摇时间为 $6 \mathrm{~h}$

Figure 8 Percentage of dye removal at different amount of magnetic MWCNTs for three nitroimidazole pharmaceuticals. Conditions: $\mathrm{pH}$ value of each pharmaceutical sample is 7.0; volume of each pharmaceutical sample is $50 \mathrm{~mL}$; the concentration of each pharmaceutical sample is $10 \mathrm{mg} \cdot \mathrm{L}^{-1}$; the agitation time is $6 \mathrm{~h}$

\section{4 吸附动力学和速度控制机理}

为了探讨磁性 MWCNTs 对混合液中硝基咪唑类药 物吸附速率的影响, 研究了不同接触时间对甲硝唑、奥 硝唑、替硝唑吸附量的影响. 图 9 给出了在 $298 \mathrm{~K}$ 下接 触时间对于三种药物在磁性 MWCNTs 上去除率的影响. 在吸附初始阶段, 三种药物在磁性 MWCNTs 上的去除 百分数随接触时间的延长而持续增大，直至在 $300 \mathrm{~min}$ 后达到吸附平衡. 这个现象归于初始阶段有大量的未被 占据的表面位点可用于吸附，一段时间后，由于溶质分 子与固体和本体相之间的排斥力使剩余的空表面位点 难以被占据.

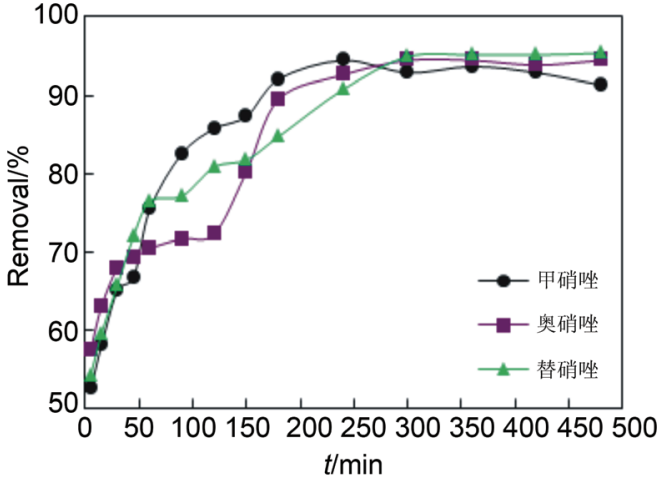

图 9 接触时间对磁性 MWCNTs 吸附药物的影响. 条件: $50 \mathrm{~mL} 10$ $\mathrm{mg} \cdot \mathrm{L}^{-1}$ 药物, $\mathrm{pH} 7$, 吸附剂量 $0.25 \mathrm{~g}$.

Figure 9 The effect of contact time on the adsorption of pharmaceuticals on magnetic MWCNTs. Conditions: $50 \mathrm{~mL}$ of $10 \mathrm{mg} \cdot \mathrm{L}^{-1}$ of pharmaceuticals, $\mathrm{pH} 7$, adsorbent dosage $0.25 \mathrm{~g}$.

\subsection{1 准一级模型与准二级模型}

为了深入了解吸附过程的特性, 将间歇实验的数据 用准一级和准二级动力学模型进行拟合 ${ }^{[24,25]}$, 其相应线 性形态如下:

$$
\begin{aligned}
& \ln \left(q_{\mathrm{e}}-q_{t}\right)=\ln \left(q_{\mathrm{e}}\right)-k_{1} t \\
& \frac{t}{q_{t}}=\frac{1}{k_{2} q_{\mathrm{e}}^{2}}+\frac{t}{q_{\mathrm{e}}}
\end{aligned}
$$

式中, $q_{\mathrm{e}}$ 与 $q_{t}\left(\mathrm{mg}^{\circ} \mathrm{g}^{-1}\right)$ 分别是平衡时与时间 $t(\mathrm{~min})$ 时单 位吸附剂吸附的药物量. $k_{1}\left(\mathrm{~min}^{-1}\right)$ 与 $k_{2}\left(\mathrm{~g} \cdot \mathrm{mg}^{-1} \cdot \mathrm{min}^{-1}\right)$ 分 别是准一级和准二级速度常数. 表 1 给出了准一级和准 二级动力学模型拟合的动力学参数和相关系数 $\left(R^{2}\right)$. 可 见准二级动力学模型的 $R^{2}$ 值远高于准一级动力学模型, 而且依据准二级模型计算的 $q_{\mathrm{e}}$ 值 $\left(q_{\mathrm{e}, \mathrm{cal}}\right)$ 与实验值 $\left(q_{\mathrm{e}, \mathrm{exp}}\right)$ 比较接近. 这意味着硝基咪唑类药物的吸附动力学服从 准二级动力学模型. 此类现象比较常见 ${ }^{[26,27]}$, 原因是在 计算准一级模型的各参数之前需要通过实验确定平衡 吸附量 $q_{\mathrm{e}}$, 而这在实际过程中很难做到. 然而准二级方 程包含了吸附的所有过程(外部夜膜扩散、表面吸附和 颗粒内扩散等), 可真实、全面地反映甲硝唑、奥硝唑、 替硝唑在固体表面的吸附机理.

\subsubsection{Weber-Morris 动力学模型}

为了确定三种硝基咪唑类化合物在吸附过程中的 实际速率控制步骤, 采用 Weber-Morris 方程 ${ }^{[28]}$ (公式 4) 对动力学数据进行分析:

$$
q_{t}=k_{\mathrm{i}} t^{1 / 2}+C
$$

式中, $k_{\mathrm{i}}$ 是粒子内扩散速度常数 $\left(\mathrm{mg} \cdot \mathrm{g}^{-1} \cdot \mathrm{min}^{-1 / 2}\right), C$ 是 反映边界层效应的常数 $\left(\mathrm{mg} \cdot \mathrm{g}^{-1}\right) \cdot q_{t}$ 对 $t^{1 / 2}$ 的图形列于图 10 , 相应的动力学参数列于表 2 .

图 10 出现多线性的图形, 表明吸附过程涉及两个 或两个以上的步骤 ${ }^{[29]}$. 初始区域发生的是扩散吸附阶 段，归于吸附质跨液膜到吸附剂外表面的扩散(外扩散). 
表 1 磁性多壁碳纳米管吸附三种硝基咪唑药品的动力学参数

Table 1 Kinetic parameters for the adsorption of three nitroimidazole pharmaceuticals onto the magnetic MWCNTs

\begin{tabular}{|c|c|c|c|c|c|c|c|c|}
\hline \multirow{2}{*}{ Adsorbate } & \multicolumn{4}{|c|}{ Pseudo first-order rate model } & \multicolumn{4}{|c|}{ Pseudo second-order rate model } \\
\hline & $q_{\mathrm{e}}($ expt. $) /\left(\mathrm{mg} \bullet \mathrm{g}^{-1}\right)$ & $q_{\mathrm{e}}($ cal. $) /\left(\mathrm{mg} \cdot \mathrm{g}^{-1}\right)$ & $k_{1} /\left(\mathrm{h}^{-1}\right)$ & $R^{2}$ & $q_{\mathrm{e}}($ expt. $) /\left(\mathrm{mg} \cdot \mathrm{g}^{-1}\right)$ & $q_{\mathrm{e}}($ cal. $) /\left(\mathrm{mg} \mathrm{g}^{-1}\right)$ & $k_{2} /\left(\mathrm{g} \cdot \mathrm{mg}^{-1} \cdot \mathrm{h}^{-1}\right)$ & $R^{2}$ \\
\hline Metronidazole & 0.4910 & 0.2805 & 0.0113 & 0.9592 & 0.4910 & 0.5017 & 0.0246 & 0.9969 \\
\hline Ornidazole & 0.4887 & 0.2812 & 0.0105 & 0.9308 & 0.4887 & 0.5163 & 0.0170 & 0.9916 \\
\hline Tinidazole & 0.6045 & 0.3607 & 0.0134 & 0.9327 & 0.6045 & 0.6254 & 0.0314 & 0.9974 \\
\hline
\end{tabular}

表 2 由 Weber-Morris 动力学模型计算的磁性多壁碳纳米管吸附硝基 咪唑类药物的动力学参数

Table 2 Kinetic parameters calculated from Weber-Morris kinetic model for nitroimidazoles adsorption on magnetic MWCNTs

\begin{tabular}{lcccccc}
\hline Adsorbate & $k_{\mathrm{i} 1}$ & $k_{\mathrm{i} 2}$ & $C_{1}$ & $C_{2}$ & $R_{1}{ }^{2}$ & $R_{2}{ }^{2}$ \\
\hline Metronidazole & 0.0288 & 0.0151 & 0.1138 & 0.2004 & 0.9617 & 0.9662 \\
Ornidazole & 0.0248 & 0.0117 & 0.1892 & 0.1892 & 0.9572 & 0.9610 \\
Tinidazole & 0.0267 & 0.0143 & 0.2704 & 0.3473 & 0.9936 & 0.9708 \\
\hline
\end{tabular}

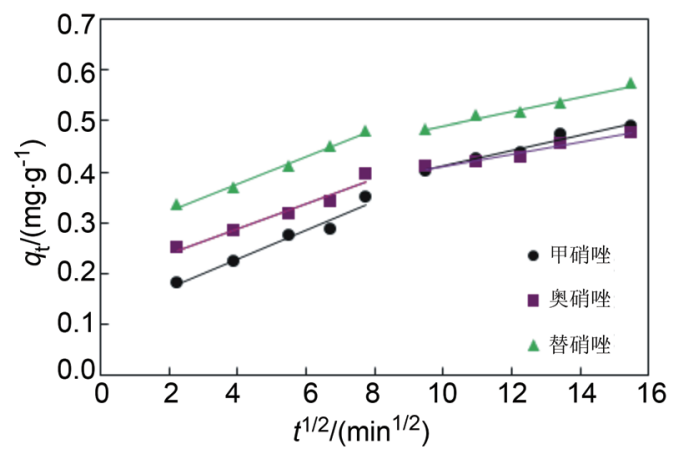

图 10 硝基咪唑类化合物在磁性 MWCNTs 上吸附的粒子内扩散图像 Figure 10 Intraparticle diffusion plots for the adsorption of the pharmaceuticals onto the magnetic MWCNTs

第二个区域是渐进的吸附阶段, 对应于吸附质从吸附剂 的外表面到达吸附剂的孔隙或内表面的毛细管扩散(内 扩散). 因此, 外扩散和颗粒内扩散过程共同影响着磁 性 MWCNTs 对甲硝唑、奥硝唑、替硝唑的吸附. 从图 10 也可看出虽然 $q_{t}$ 对 $t^{1 / 2}$ 的回归为线性, 但曲线没有通 过原点, 因此, 颗粒内扩散并不是唯一的速率限制步骤, 而是由外扩散和粒子内扩散共同控制 ${ }^{[30]}$.

\subsubsection{Boyd 动力学模型}

为获得吸附过程中药物吸附准确的速度控制步骤, 采用 Boyd 动力学模型对吸附动力学数据进行进一步的 分析，该模型可表示如下 ${ }^{[31]}$ :

$$
F=1-\frac{6}{\pi^{2}} \exp \left(-B_{t}\right)
$$

式中, $F$ 是不同时间 $t$ 时被吸附的溶质部分, $B_{t}$ 是 $F$ 的数 学函数, $F$ 由下式给出:

$$
F=\frac{q_{t}}{q_{\mathrm{e}}}
$$

结合方程(5)与方程(6), 动力学表达式转换如下:

$$
B_{t}=-\ln \left(1-\frac{q_{t}}{q_{e}}\right)-0.4977
$$

图 11 给出了 $B_{t}$ 值对时间 $t$ 绘制的图形. 将该图形 线性化可提供区分有关吸附控制机理的外质量传递与 粒子内扩散的有用信息 ${ }^{[32]}$.

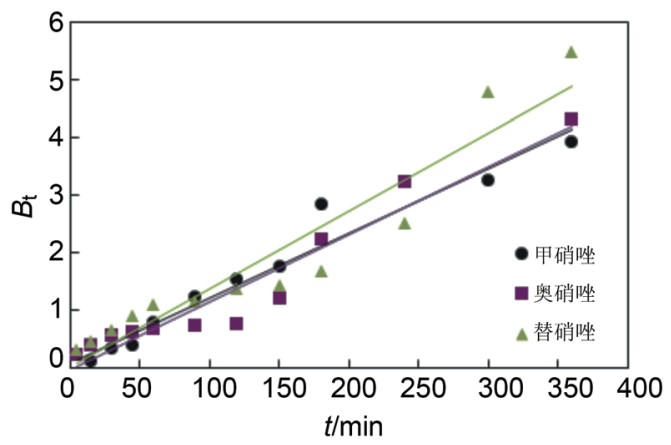

图 11 甲硝唑、奥硝唑、替硝唑吸附到磁性 MWCNTs 上的 Boyd 图 Figure 11 Boyd plots for the adsorption of three pharmaceuticals onto the magnetic MWCNTs

如图所示, 线性化图形的起点虽然离原点较近但该 线段并不通过原点，意味着吸附过程涉及到外质量传 递 $^{[33]}$, 但这些线段也不是真正意义上的直线, 说明外质 量传递对于三种硝基咪唑类药物在磁性 MWCNT 上的 吸附是相当弱的速率控制步骤。这个结果与上述 Weber-Morris 动力学研究中的吸附速率控制机理有着 相似之处.

\section{5 吸附等温线}

吸附等温线描述了溶质是如何与吸附剂发生相互 作用的. 甲硝唑在 MWCNT 上的平衡吸附量作为甲硝 唑平衡浓度的函数，绘制在图 12 中. 可看出甲硝唑的平 衡吸附量在 $308 \mathrm{~K}$ 时最低, 在 $288 \mathrm{~K}$ 时最高, 说明降低 温度有利于甲硝唑在磁性 MWCNTs 上的吸附.

常用 Langmuir 模型和 Freundlich 模型描述平衡吸附 数据. Langmuir 等温线模型假定吸附表面为均相(所有 吸附位点具有相同的吸附亲合势 $)^{[34]}$, 而 Freundlich 等温 线是关于非均相表面的吸附经验关系式 ${ }^{[35]}$. Langmuir 与 Freundlich 吸附等温线模型表示如下:

$$
\frac{C_{\mathrm{e}}}{q_{\mathrm{e}}}=\frac{C_{\mathrm{e}}}{q_{\mathrm{m}}}+\frac{1}{b q_{\mathrm{m}}}
$$




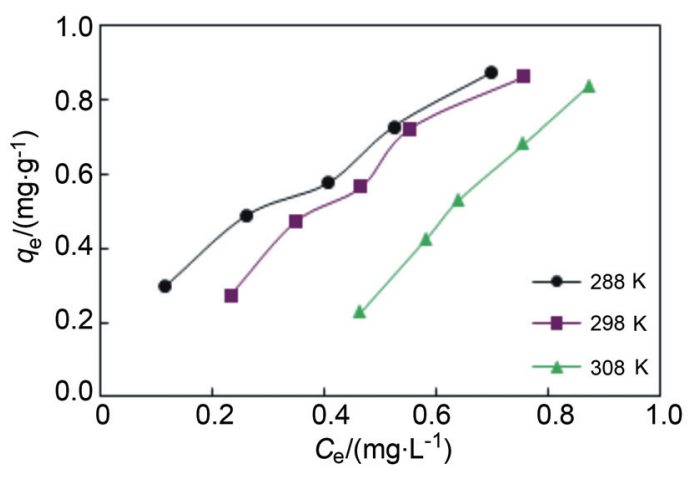

图 12 磁性 MWCNTs 吸附甲硝唑药物的等温线

Figure 12 Isotherms for the adsorption of metronidazole pharmaceuticals onto the magnetic MWCNTs

$$
\ln q_{\mathrm{e}}=\ln K_{f}+\frac{1}{n} \ln C_{\mathrm{e}}
$$

式中, $q_{\mathrm{m}}$ 是最大的单层吸附容量 $\left(\mathrm{mg}^{\circ} \mathrm{g}^{-1}\right) ; C_{\mathrm{e}}$ 是甲硝唑的 平衡浓度 $\left(\mathrm{mg} \cdot \mathrm{L}^{-1}\right) ; q_{\mathrm{e}}$ 是平衡浓度下单位重量的磁性 MWCNTs 对甲硝唑的吸附量 $\left(\mathrm{mg}^{\circ} \mathrm{g}^{-1}\right) ; b$ 是 Langmuir 常 数 $\left(\mathrm{L} \cdot \mathrm{mg}^{-1}\right)$, 它与结合位点的亲和性相关. Freundlich 等温线常数可从温度 $288,298,308 \mathrm{~K}$ 下 $q_{\mathrm{e}}$ 对 $C_{\mathrm{e}}$ 的图形 确定; 参数 $K_{\mathrm{f}}$ 是吸附容量的大致指标 $\left(\mathrm{L} \cdot \mathrm{mg}^{-1}\right), n$ 是吸附 强度 $\left(\mathrm{g} \cdot \mathrm{L}^{-1}\right)$. 表 3 给出了在 $298 \mathrm{~K}$ 下将吸附平衡数据拟 合于 Langmuir 和 Freundlich 模型得到的参数(288 和 308 $\mathrm{K}$ 下的参数值与 $298 \mathrm{~K}$ 下的参数有类似趋势，故省略).

根据表 3 中的数据, Freundlich 模型的相关系数 $\left(R^{2}\right)$ 位于 $0.97 \sim 0.98$ 之间, 明显大于 Langmuir 模型的 $R^{2}$ 值. 所以 Freundlich 模型比 Langmuir 模型更好地描述了甲硝 唑、奥硝唑、替硝唑在磁性 MWCNTs 上的吸附.

对于等温过程, Langmuir 模型倾向于描述单分子层 吸附, 即每个吸附位点只吸附一个吸附质微粒, 当所有 吸附位置都被占据后就达到动态吸附平衡. 由此可推断 水中甲硝唑、奥硝唑、替硝唑在磁性 MWCNTs 上的吸 附机理并不倾向于单分子层吸附, 而是涉及到更为复杂 的吸附机理. 在 Freundlich 模型中, 常数 $n$ 与吸附推动 力的强弱有关, 表 2 中 $n$ 的值由大变小也说明降低温度 对吸附有利.

分离因子(式(10))是一个与 Langmuir 等温线基本特 性有关的无量纲的常数,

$$
R_{\mathrm{L}}=\frac{1}{1+b C_{0}}
$$

式中, $C_{0}$ 是吸附质的最大初始浓度 $\left(\mathrm{mg} \cdot \mathrm{L}^{-1}\right), b\left(\mathrm{~L} \cdot \mathrm{mg}^{-1}\right)$ 是 Langmuir 吸附常数. 在 288,298 和 $308 \mathrm{~K}$ 下, 对应的 $R_{\mathrm{L}}$ 值都介于 0 到 1 之间，表明三种化学品的吸附是有利 的. $R_{\mathrm{L}}$ 值反映吸附等温线的形状，表示在给定吸附质的 浓度范围内, 某吸附剂对吸附质发生吸附现象的可行 性. 例如, 当 $R_{\mathrm{L}}>1$ 时表示不利于吸附; $0<R_{\mathrm{L}}<1$ 表示 可以进行; $R_{\mathrm{L}}=1$ 表示吸附过程是线性的; 而 $R_{\mathrm{L}}=0$ 表示 吸附过程是不可逆的.

\section{6 吸附热力学}

从下列方程式计算热力学参数:

$$
\Delta G^{0}=-R T \ln K_{\mathrm{c}}
$$

式中, $R$ 是普适气体常数 $\left(8.314 \mathrm{~J} \cdot \mathrm{mol}^{-1} \cdot \mathrm{K}^{-1}\right), T$ 是热力学 温度 $(K), K_{\mathrm{c}}$ 是分布系数. 吸附过程的吉布斯自由能变量 $\left(\Delta G^{0}\right)$ 可由不同温度下的 $\ln K_{\mathrm{c}}$ 值计算, 而 $K_{\mathrm{c}}$ 值则由以下 方程式计算:

$$
K_{\mathrm{c}}=\frac{q_{\mathrm{e}}}{C_{\mathrm{e}}}
$$

式中, $C_{\mathrm{e}}$ 是吸附平衡状态下的吸附质的浓度; $q_{\mathrm{e}}$ 是在平 衡浓度下单位量的 MWCNTs 吸附硝基咪唑类药物的质 量 $\left(\mathrm{mg} \bullet \mathrm{g}^{-1}\right)$.

吸附过程的焓变量 $\left(\Delta H^{0}\right)$ 和熵变量 $\left(\Delta S^{0}\right)$ 由以下方程 式计算:

$$
\ln K_{\mathrm{c}}=\frac{\Delta S^{0}}{R}-\frac{\Delta H^{0}}{R T}
$$

根据方程式(13), 将 $\ln K_{\mathrm{c}}$ 对 $1 / T$ 绘图, 由所得直线 的斜率与截距分别计算出 $\Delta H^{0}$ 和 $\Delta S^{0}$ 参数.

热力学参数列在表 4 中. 负 $\Delta G^{0}$ 表明吸附过程的自 发性. 正的 $\Delta S^{0}$ 值表明三种硝基咪唑类药物的吸附是一 个熵增的过程，这可能与吸附过程中伴随着大量水分子 从 MWCNTs 表面解吸的现象有关 ${ }^{[36]}$. 负 $\Delta H^{0}$ 表明吸附 过程是放热的, 这个现象与三种药物在磁性 MWCNTs 上的吸附是随温度升高而下降是一致的(图 10). 在一般 情况下, 可由 $\Delta G^{0}$ 值评估吸附过程的性质, $\Delta G^{0}$ 值在 0 至一 $20 \mathrm{~kJ} \cdot \mathrm{mol}^{-1}$ 范围是物理吸附; 而化学吸附的 $\Delta G^{0}$ 值 则位于 -80 至 $-400 \mathrm{~kJ} \cdot \mathrm{mol}^{-1[37]}$. 根据本研究中的 $\Delta G^{0}$ 值, 可判断三种硝基咪唑类药物在 MWCNTs 上的吸附 过程主要是物理吸附.

\section{7 吸附稳定性}

\begin{tabular}{|c|c|c|c|c|c|c|}
\hline \multirow{2}{*}{ Adsorbate } & \multicolumn{3}{|c|}{ Langmuir model } & \multicolumn{3}{|c|}{ Freundlich model } \\
\hline & $q_{\mathrm{m}} /\left(\mathrm{mg} \bullet^{-1}\right)$ & $b /\left(\mathrm{L} \cdot \mathrm{mg}^{-1}\right)$ & $R^{2}$ & $K_{\mathrm{f}} /\left(\mathrm{mg} \bullet \mathrm{g}^{-1}\right)$ & $n /\left(\mathrm{g} \cdot \mathrm{L}^{-1}\right)$ & $R^{2}$ \\
\hline Metronidazole & 0.7824 & 3.4949 & 0.6217 & 1.2070 & 1.0272 & 0.9747 \\
\hline Ornidazole & 0.4607 & 8.1959 & 0.9653 & 1.1935 & 0.8610 & 0.9897 \\
\hline Tinidazole & 0.7444 & 1.7357 & 0.9644 & 1.1551 & 1.1744 & 0.9871 \\
\hline
\end{tabular}

金属离子从吸附剂中的泄漏是不希望发生的，所以 在不同 $\mathrm{pH}$ 水溶液中测试了 $\mathrm{Fe}$ 的溢出情况，以评估磁性

表 3 三种硝基咪唑类药物在磁性 MWCNTs 上的吸附等温线参数

Table 3 Adsorption isotherms parameters of three nitroimidazoles onto magnetic MWCNTs 
表 4 不同温度下磁性多壁碳纳米管吸附水溶液中三种硝基咪唑类药 物的热力学参数

Table 4 The thermodynamic parameters of the three nitroimidazoles adsorbed onto the magnetic MWCNTs under different temperature

\begin{tabular}{ccccccc}
\hline & \multirow{2}{*}{ Adsorbate } & $\Delta H^{0} /$ & $\Delta S^{0} /$ & \multicolumn{3}{c}{$\Delta G^{0} /\left(\mathrm{kJ} \bullet \mathrm{mol}^{-1}\right)$} \\
\cline { 3 - 7 }$\left(\mathrm{kJ} \bullet \mathrm{mol}^{-1}\right)$ & $\begin{array}{c}\left(\mathrm{J} \bullet \mathrm{mol}^{-1}\right. \\
\left.\mathrm{K}^{-1}\right)\end{array}$ & $288 \mathrm{~K}$ & $298 \mathrm{~K}$ & $308 \mathrm{~K}$ \\
\hline Metronidazole & -28.482 & 1.2604 & -28.845 & -28.858 & -28.870 \\
Ornidazole & -12.222 & 16.713 & -17.035 & -17.202 & -17.369 \\
Tinidazole & -11.597 & 20.693 & -17.557 & -17.764 & -17.971 \\
\hline
\end{tabular}

MWCNTs 的稳定性. 图 13 给出了不同 $\mathrm{pH}$ 下溢出 $\mathrm{Fe}$ 的 浓度.

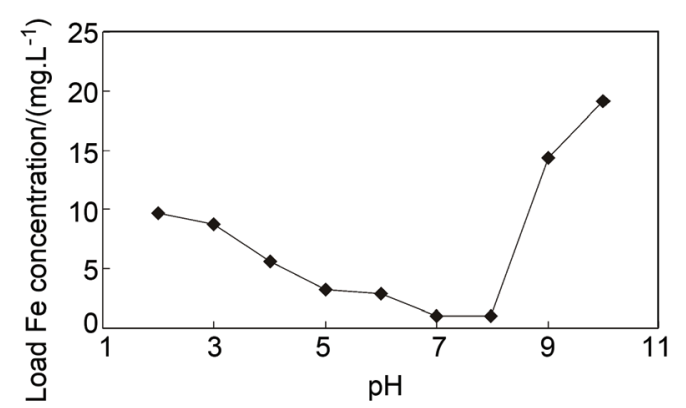

图 13 不同 $\mathrm{pH}$ 条件下磁性 MWCNTs 中铁的泄露

Figure 13 Leached Fe content of magnetic MWCNTs under different $\mathrm{pH}$ levels

从图 13 可发现在 $\mathrm{pH} 7$ 及附近铁的溢出很少, 而当 $\mathrm{pH}$ 低于 4 或大于 8 则铁的溢出大为增加. 以中性 $\mathrm{pH}$ 为 例, 水中磁性 MWCNTs 溢出的铁离子的量是 $0.96 \mathrm{mg}$ $\mathrm{L}^{-1}$. 此现象与先前研究观测的铁离子从 $\mathrm{Fe}_{3} \mathrm{O}_{4}$ 复合物的 溢出显示出类似的趋势 ${ }^{[38]}$. 根据水中溢出铁的量和总 铁的量(相关计算过程未给出), 可计算出中性 $\mathrm{pH}$ 条件 下磁性 MWCNTs 中 $\mathrm{Fe}$ 溢出约占总 $\mathrm{Fe}$ 的百分比是 $0.0869 \%$. 这个结果说明吸附剂中铁的溢出是可以忽略 不计的.

\section{4 结论}

在制备磁性 MWCNTs 基础上, 用 TEM、XRD、FTIR 分析方法对吸附剂的结构进行了表征, 证实磁化后的 MWCNTs 含有羧基官能团, 而且 MWCNTs 的表面和管 内包含了 $\mathrm{Fe}_{3} \mathrm{O}_{4}$ 纳米颗粒. 制备的磁性吸附剂可以很好 地分散在水中, 并且在吸附后可容易地由外磁场从介质 中分离. 磁性 MWCNTs 对三种底物的有效吸附主要源 于碳纳米管独特的多壁结构和壳层上的羧基, 它们对于 吸附硝基咪唑类药物分子提供了大的表面积和良好的 亲和力. 药物的吸附依赖于 $\mathrm{pH}$ 值; 当溶液 $\mathrm{pH}$ 值为中性 时, 去除率最大. 动力学研究表明, 在 $300 \mathrm{~min}$ 达到了吸 附平衡，此时三种硝基咪唑类药物的吸附率都达到 $90 \%$ 以上, 吸附过程服从准二级模型, 吸附的总体速度受到 外质量传递和粒子内扩散二者的影响, 但主要受后者的
控制. 吸附等温线可很好地拟合 Freundlich 吸附等温线 方程. 热力学参数表明甲硝唑、奥硝唑、替硝唑在磁性 MWCNTs 上的吸附是自发、放热和物理的吸附过程. 吸 附机理可能是硝基咪唑类药物与 MWCNTs 之间的静电 引力和 $\pi-\pi$ 堆积作用. 本研究的结果表明, 磁性 MWCNTs 具备去除水中硝基咪唑类药物的潜在应用性.

\section{References}

[1] Wang, Y. Q.; Zhang, P. P.; Jiang, N. L.; Gong, X. J.; Meng, L.; Wang, D. W.; Qu, N.; Zhang, H. B. J. Chromatogr. B 2012, 899, 27.

[2] Tan, H. Y.; Yang, G. P.; Yu, P.; Huang, Y. Y.; Pei, Q.; Yuan, H.; Huang, Z. J.; Xie, F. F.; Mu, L. L. J. Pharm. Biomed. Anal. 2013, 76,75 .

[3] Xie, Z. Y.; Zhao, Y. G.; Sun, D. Y.; Zhou, L.; Wang, W. W.; Song, H.; Wu, W. J. Clin. J. Med. Offic. 2012, 40, 341. (谢子英, 赵亚刚, 孙大勇, 周兰, 王卫卫, 宋鸿, 吴炜景, 临床军医杂志, 2012, 40, 341.)

[4] Fang, Z. H.; Chen, J. H.; Qiu, X. H.; Qiu, X. Q.; Cheng, W.; Zhu, L. C. J. Desalination 2011, 268, 60.

[5] Zhang, Y.; Yue, Z. F.; Lan, F.; Zhao, F. J.; Xiao, C. G.; Ou Yang, S.; Wu, W. D.; Wu, Y. N.; Li, L. S. Chin. J. Anal. Chem. 2012, 40, 724. (张毅, 岳振峰, 蓝芳, 赵风娟, 肖陈贵, 欧阳姗, 吴卫东, 吴 永宁, 李丽苏, 分析化学, 2012, 40, 724.)

[6] Chen, J. H.; Qiu, X. Q.; Fang, Z. Q.; Yang, M.; Tsang, P.; Gu, F. L.; Cheng, W.; Lan, B. Y. Chem. Eng. J. 2012, 181-182, 113.

[7] Vertzoni, M.; Carlsson, A.; Abrahamsson, B.; Goumas, K.; Reppas, C. Int. J. Pharm. 2011, 413, 81.

[8] Yu, K.; Li, B.; Zhang, T. Anal. Chim. Acta 2012, 738, 59.

[9] Zhou, X. F.; Zhang, Y. L.; Dai, C. M. J. Environ. Prot. Sci. 2009, 35, 15. (周雪飞, 张亚雷, 代朝猛, 环境保护科学, 2009, 35, 15.)

[10] Zhang, J. B.; Zhou, Q. F.; Liu, W.; Jiang, G. B. J. Environ. Sci. 2009, 29, 1056. (张建斌, 周群芳, 刘伟, 江桂斌, 环境科学学报, 2009, 29, 1056.)

[11] Zhang, L.; Song, X. Y.; Liu, X. Y.; Yang, L. J.; Pan, F. Chem. Eng. J. 2011, 178, 26.

[12] Yavuz, C. T.; Mayo, J. T.; Yu, W. W.; Prakash, A.; Falkner, J. C.; Yean, S.; Cong, L.; Shipley, H. J.; Kan, A.; Tomson, M.; Natelson, D.; Colvin, V. L. Science 2006, 314, 964.

[13] Liu, J. F.; Zhao, Z. S.; Jiang, G. B. Environ. Sci. Technol. 2008, 42, 6949.

[14] Gong, J. L.; Wang, B.; Zeng, G. M.; Yang, C. P.; Niu, C. G.; Niu, Q. Y.; Zhou, W. J.; Liang, Y. J. Hazard. Mater. 2009, 164, 1517.

[15] Ai, L. H.; Zhang, C. Y.; Liao, F.; Wang, Y.; Li, M.; Meng, L. Y.; Jiang, J. J. J. Hazard. Mater. 2011, 198, 282.

[16] Xiong, Z. H.; Wang, L.; Zhou, J. G.; Liu, J. M. Acta Phys.-Chim. Sin. 2010, 26, 2890. (熊振湖, 王璐, 周建国, 刘建明, 物理化学学 报, 2010, 26, 2890.)

[17] Tayyebeh, M.; Abbas, A.; Mazaher, A.; Hasan, B. J. Hazard. Master. 2011, 196, 109.

[18] Zhang, Q.; Zhu, M. F.; Zhang, Q. H.; Li, Y. G.; Wang, H. Z. Compos. Sci. Technol. 2009, 69, 638.

[19] Luo, X. B.; Zhan, Y. C.; Huang, Y. N.; Yang, L. X.; Tu, X. M.; Luo, S. L. J. Hazard. Mater. 2011, 187, 274.

[20] Qi, J. Y. Modern Analytical and Test Techniques, Tongji University Press, Shanghai, 2006, p. 332. (祁景玉，现代分析测试技术，同济 大学出版社, 上海, 2006, p. 332.)

[21] Lin, Z. K.; Cheng, W. J.; Li, Y. Y.; Liu, Z. R.; Chen, X. P.; Huang, C. J. Anal. Chim. Acta 2012, 720, 71.

[22] Wang, X.; Wang, L. Y.; He, X. W.; Zhang, Y. K.; Chen, L. X. Talanta 2009, 78, 332.

[23] Xing, Q. Y.; Pei, W. W.; Xu, R. Q.; Pei, J. Basic Organic Chemistry, Higher Education Press, Beijing, 2005, p. 899. (邢其毅, 裴伟伟, 徐瑞秋, 裴坚, 基础有机化学, 高等教育出版社, 北京, $2005, \mathrm{p}$. 899.)

[24] Zhang, L. W.; Petersen, E. J.; Zhang, W.; Chen, Y. S.; Cabrera, M.; Huang, Q. G. Environ. Pollut. 2012, 166, 75.

[25] Saleh, T. A.; Agarwal, S.; Gupta, V. K. Appl. Catal., B: Environ. 2011, 106, 46.

[26] Fasfous, I. I.; Radwan, E. S.; Dawoud, J. N. Appl. Surf. Sci. 2010 $256,7246$. 
[27] Arasteh, R.; Masoumi, M.; Rashidi, A. M.; Moradi, L.; Samimi, V.; Mostafavi, S. T. Appl. Surf. Sci. 2010, 256, 4447.

[28] Shi, Y.; Kong, X. Z.; Zhang, C. M.; Chen, Y. M.; Hua, Y. F. Chem. Eng. J. 2013, 215-216, 113.

[29] Chen, G. C.; Shan, X. Q.; Zhou, Y. Q.; Shen, X. E.; Huang, H. L.; Khan, S. U. J. Hazard. Mater. 2009, 169, 912.

[30] Badriya, A. R.; Chedly, T.; Nidal, H. Chem. Eng. J. 2012, 183, 294.

[31] Nakorn, S.; Patiparn, P.; Chawalit, N. Chem. Eng. J. 2013, 214, 208.

[32] Nancy, Y. A.; Sol, M. M.; Fanor, M.; Elizabeth, F. Comput. Theor. Chem. 2013, 1005, 16.
[33] Somayeh, B.; Mehran, S.; Hossein, S. Microporous Mesoporous Mater. 2013, 168, 30.

[34] Zhang, P. K.; Li, W. Sep. Purif. Technol. 2010, 70, 367.

[35] Gautham, P. J.; Clement, T. P. J. Contam. Hydrol. 2012, 129-130, 46.

[36] Kumar, A. K.; Mohan, S. V. Sep. Purif. Technol. 2012, 87, 22.

[37] Kuo, C. Y.; Wu, C. H.; Wu, J. Y. J. Colloid Interface Sci. 2008, 327, 308.

[38] Inbaraj, B. S.; Chen, B. H. Bioresour. Technol. 2011, 102, 8868. 